\title{
The alexithymic brain: the neural pathways linking alexithymia to physical disorders
}

\author{
Michiko Kano* and Shin Fukudo
}

\begin{abstract}
Alexithymia is a personality trait characterized by difficulties in identifying and describing feelings and is associated with psychiatric and psychosomatic disorders. The mechanisms underlying the link between emotional dysregulation and psychosomatic disorders are unclear. Recent progress in neuroimaging has provided important information regarding emotional experience in alexithymia. We have conducted three brain imaging studies on alexithymia, which we describe herein. This article considers the role of emotion in the development of physical symptoms and discusses a possible pathway that we have identified in our neuroimaging studies linking alexithymia with psychosomatic disorders. In terms of socio-affective processing, alexithymics demonstrate lower reactivity in brain regions associated with emotion. Many studies have reported reduced activation in limbic areas (e.g., cingulate cortex, anterior insula, amygdala) and the prefrontal cortex when alexithymics attempt to feel other people's feelings or retrieve their own emotional episodes, compared to nonalexithymics. With respect to primitive emotional reactions such as the response to pain, alexithymics show amplified activity in areas considered to be involved in physical sensation. In addition to greater hormonal arousal responses in alexithymics during visceral pain, increased activity has been reported in the insula, anterior cingulate cortex, and midbrain. Moreover, in complex social situations, alexithymics may not be able to use feelings to guide their behavior appropriately. The lowa gambling task (IGT) was developed to assess decision-making processes based on emotion-guided evaluation. When alexithymics perform the IGT, they fail to learn an advantageous decision-making strategy and show reduced activity in the medial prefrontal cortex, a key area for successful performance of the IGT, and increased activity in the caudate, a region associated with impulsive choice. The neural machinery in alexithymia is therefore activated more on the physiologic, motor-expressive level and less in the cognitive-experiential domains of the emotional response system. Affects may play an important role in alleviating intrinsic physiologic reactions and adapting to the environment. Deficient development of emotional neural structures may lead to hypersensitivity to bodily sensations and unhealthy behaviors, a possible mechanism linking alexithymia to psychosomatic disorders.
\end{abstract}

Keywords: Affect, Alexithymia, Emotional dysregulation, Neuroimaging, Psychosomatic disorders

\section{Introduction}

The alexithymia concept was introduced by Sifneos [1] from the clinical observation of patients with classic psychosomatic diseases, in which they failed to respond to dynamic psychotherapy. It was conceptualized as encompassing a cluster of cognitive traits including difficulty identifying feelings, difficulty describing feelings to others, externally oriented thinking, and a limited imaginative capacity [2]. Research investigating the alexithymia construct has advanced rapidly, owing to the

\footnotetext{
* Correspondence: kano-m@med.tohoku.ac.jp

Behavioral Medicine, Tohoku University Graduate School of Medicine, Tohoku University, 2-1 Seiryo-cho, Aoba-ku, Sendai 980-8575, Japan
}

development of the self-reported, 20-item, Toronto Alexithymia Scale (TAS-20) [3,4]. The TAS-20 provides investigators with a reliable, validated, and common metric for measuring the construct [5,6]. Individuals with a TAS-20 total score of $\geq 61$ are considered alexithymic, and those with a score of $<51$ are considered nonalexithymic [7]. The TAS-20 comprises three subscales: difficulty identifying feelings, difficulty describing one's feelings, and presence of externally oriented thinking $[5,6]$. Alexithymia was assessed by the TAS-20 in the studies addressed in this article. However, we note some researchers question whether self-report measures of alexithymia, including the TAS-20, provide a valid assessment
C Biomed Central

(c) 2013 Kano and Fukudo; licensee BioMed Central Ltd. This is an Open Access article distributed under the terms of the Creative Commons Attribution License (http://creativecommons.org/licenses/by/2.0), which permits unrestricted use, distribution, and reproduction in any medium, provided the original work is properly cited. 
of the construct and recommend the use of multiple methods of measurement $[8,9]$. Various instruments have been developed, such as observer-rated measures including the modified Beth Israel Hospital Psychosomatic Questionnaire (BIQ), the Observer Alexithymia Scale, a set of Rorschach variables and the Toronto Structured interview for Alexithymia [10]. The Levels of Emotional Awareness Scale (LEAS) is also a self-report instrument to elicit written emotional response and often compared to the TAS-20 [11].

Although alexithymia is personality trait independent from clinical diseases, a high rate of alexithymia has been reported in several medical conditions and psychiatric disorders such as asthma, hypertension, and chronic pain [5,6]. In particular, it is an important phenomenon in a relevant subgroup of patients with functional somatic disorders including functional gastrointestinal disorders [12-15]. It is also associated with increased mortality $[16,17]$. The prevalence of alexithymia is approximately $10 \%$ in the general population, whereas a high rate of alexithymia (approximately 40-60\%) is reported among patients with psychosomatic disorders $[7,12]$. Alexithymia is considered a key element associated with the psychosomatic process $[18,19]$ and appears to play a role in neurologic causes of physical illness [20].

The mechanism underlying alexithymia and its association with physical illness remain to be elucidated. Nevertheless, several pathways have been suggested, as follows: alexithymia leads to organic disease via physiologic or behavioral mechanisms; alexithymia leads to illness behavior (physical symptoms, disability, and excessive health care use) via cognitive or social mechanisms; physical illness leads to alexithymia; both alexithymia and physical illness result from sociocultural or biologic factors [20].

Physiologically, because the main problem of alexithymic personality traits is considered to be deficit in affect regulation, it is hypothesized that alexithymia constitutes a longstanding risk factor for imbalances in the autonomic nervous system and the neuroendocrine system [15]. The few immunology studies that have been conducted have found that alexithymia is associated with poor immune status [5], and a number of studies have found that alexithymia is related to increased levels of resting (tonic) sympathetic or cardiovascular activity [5]. Recent progress in neuroimaging studies on alexithymia has provided important information on the neural basis of alexithymia. In particular, a number of studies have examined brain responses underlying affect dysregulation in alexithymia [21-36]. In addition to dysfunction of the autonomic nervous system and neuroendocrine system, we now consider that the alexithymic brain may cause a variety of psychosomatic disorders. Here we review recent neuroimaging findings related to emotional experience in alexithymia, including those from three of our own studies on emotional processing, visceral processing, and emotion-guided decision-making, and discuss a possible neural mechanism linking alexithymia and psychosomatic processes. In contrast to the many studies focused on the personality dimensions of alexithymia, we would like to provide a unique review of alexithymia from the view point of the linking alexithymia to somatic conditions.

\section{Socio-affective processing in alexithymia}

Research has repeatedly shown impairment of emotional processing in alexithymia, that is, in the recognition of basic emotions associated with emotionally valenced sentences, words, faces, and scenes [37-39]. Therefore, in our first experiment we conducted a brain imaging study of facial expression processing in alexithymia, in which regional cerebral blood flow (rCBF) was measured by positron emission tomography (PET) [22]. Subjects performed gender discrimination of angry, sad, and happy facial expressions, as well as neutral faces, during PET scanning; thus, the emotional component of stimuli was assessed implicitly. Alexithymics exhibited lower $\mathrm{rCBF}$ in the right hemisphere in the inferior and middle frontal cortex, orbitofrontal cortex, superior temporal gyrus, inferior parietal cortex, and fusiform gyrus compared to nonalexithymics. In addition, alexithymics showed higher rCBF in the left hemisphere in the superior frontal cortex, inferior parietal cortex, superior temporal gyrus, and cerebellum compared to nonalexithymics. There was no significant difference in $\mathrm{rCBF}$ between alexithymics and nonalexithymics when they looked at neutral faces. Moreover, the dorsal anterior cingulate cortex (dACC) and bilateral insula were less activated in alexithymics in response to angry faces compared to their responses to neutral faces. The process underlying the recognition of emotion in faces has been characterized [40]. Lateral portions of the inferior occipital gyrus, fusiform gyrus, and superior temporal gyrus are disproportionately activated in facial processing [40]. The amygdala is particularly activated in response to facial expressions of fear, and the orbitofrontal cortex and ACC are activated by facial expressions of anger [40]. The right primary and secondary somatosensory areas, insula, and anterior supramarginal gyrus are involved in the recognition of emotion [40].

Therefore, our above findings indicate that alexithymics show dampened responses in emotional facial processing regions in the right hemisphere and increased responses in those areas in the left hemisphere. In addition, the dACC has been suggested to play a crucial role in conscious awareness of emotion [41,42], and dysfunction in the $\mathrm{dACC}$ has been proposed to be one of the main mechanisms of alexithymia [8]. The anterior insula appears to be critically involved in subjective emotional experience and awareness of the internal bodily state [43-46]. Hence, 
in particular, alexithymia shows less activation in brain areas associated with emotional awareness during the viewing of facial expressions.

With regard to socio-affective processing, a number of studies have demonstrated different brain responses in areas associated with emotional processing. For example, activity in the amygdala is reduced in alexithymics during the viewing of a masked, sad face [31,34], in viewing pictures evoking anxiety and disgust [47], in response to threatening emotional actions [30], and with respect to positive autobiographic recall [29]. In addition, it has been reported that a female cocaine user showed a negative correlation between activity in the right amygdala and alexithymia score in script-guided imagery of neutral or stressful situations [48]. Reports of individuals with high-functioning autism/Asperger syndrome also demonstrate that alexithymia score is negatively correlated with activity in the left amygdala during the rating of affective pictures [49]. The amygdala plays a central role in several aspects of emotional processing, such as the recognition of both positively and negatively valenced emotional stimuli [50,51]. Less activation in the insula is also reportedly associated with alexithymia in response to a masked, sad face [31] and in response to affective pictures among individuals with autism [49]. Many studies have reported alteration in activity in the ACC during socio-affective tasks. Among these, $50 \%$ report a negative correlation, $40 \%$ report a positive correlation, and $10 \%$ show both types of associations (ie, high-alexithymia individuals showed more activation of the rostral ACC, whereas low-alexithymia individuals showed more activation of the dACC) [30]. Activity in the dACC has been reported to be increased in response to implicit presentation of facial expressions [52], in contrast to our results. These discrepancies may be related to experimental variables including tasks and stimuli. For example, higher activation in the $\mathrm{dACC}$ and lower activation in the amygdala has been shown to be associated with alexithymia in the same experiment. Depending on the context of the socio-affective task, dACC activity may increase or decrease. The function of the dACC may not always be associated with emotional awareness; sometimes the ACC interacts with other parts of the brain to sustain self-regulation of one's own emotional state. The above, taken together with our results, provide accumulating evidence for dysfunction in regions related to socio-affective processing in alexithymia.

\section{Interoceptive arousal in alexithymia Somatosensory amplification}

Another well-reported characteristic of alexithymia is somatosensory amplification. Research has shown that patients with chronic pain have higher levels of alexithymia than control subjects, and alexithymia is associated with overreporting of physical symptoms [13,20,53-55]. An association between alexithymia and increased pain intensity and sensitivity to experimentally induced pain has been demonstrated [56-58]. The degree of alexithymia correlates with the score of somatosensory amplification [59]. Therefore, alexithymics might perceive signals from the body in an aberrant manner such that lowintensity stimulation is perceived as high intensity. In particular, functional gastrointestinal disorders, such as irritable bowel syndrome, characterized by chronic gastrointestinal symptoms with no biochemical abnormalities, are associated with alexithymia $[14,60,61]$.

Therefore, in our second study, we investigated brain responses to visceral stimulation induced by colonic distension between alexithymics and nonalexithymics. During colonic distension, greater activation was observed in the pregenual ACC, right insula, and midbrain in subjects with alexithymia [19]. The TAS-20 score correlated positively with both activity in the right insula and orbital gyrus and adrenaline levels in the blood in response to stimulation. Subjects with high scores for difficulty identifying feelings perceived strong pain, urgency for defecation, stress, and anxiety.

Visceral stimulation, especially by colonic distension, has been reported to elicit activation of various brain areas including the posterior insula, prefrontal cortex (PFC), ACC, and brainstem periaqueductal gray (PAG) [62-64]. A detailed, dynamic representation of particular internal bodily states and the mapping of each interoceptive state occurs within the orbitofrontal and right insular cortices $[65,66]$. In the cingulate cortex, the pregenual ACC responds to visceral stimulation and is associated with the perception of secondary pain, which is characterized by greater unpleasantness [67]. Electrical stimulation of the insula has been reported to elicit changes in blood pressure, heart rate, respiration, gastric motility, peristaltic activity, salivation, and adrenaline secretion [68]. In addition, the orbitofrontal cortex has been shown to receive robust sensory input and to act as an internal environmental integrator that coordinates behavioral, autonomic, and endocrine responses [69]. The brainstem is known to control ascending nociceptive input and nuclei such as the rostral ventromedial medulla and PAG, which are able to both inhibit and facilitate nociceptive responses. In particular, activation of the right PAG has been reported to correlate with anxiety during visceral stimulation but not somatic stimulation [70]. Therefore, it may be assumed that activation of brain areas associated with alexithymia represents afferent representation of bodily states and efferent autonomic and endocrine responses that accompany it. Alexithymics may be more aroused by interoception of unpleasant feelings than nonalexithymics, thereby displaying more autonomic responses. 
The evidence supports the somatosensory amplification hypothesis of alexithymia. There have been no other imaging studies investigating pain or interoception processing in alexithymia. However, a few, in which interoceptive or physiologic arousal was induced, have provided suggestive findings. Moriguchi et al. examined brain responses of alexithymics during visual perception of pictures depicting human hands and feet in painful situations [26]. In this task assessing empathy to others' pain, alexithymics showed greater brain activity in the posterior and anterior insula. The posterior insula is the primary endpoint of the interoceptive pathway, which represents sensory aspects from the body including pain, touch, and visceral sensation $[43,65]$. The information in the posterior insula is rerepresented in the right anterior insular cortex, which provides subjective awareness of the feelings from the body, that is, metarepresentation of interoception $[43,65]$. Other brain areas in the pain matrix, such as the dorsolateral PFC, dorsal pons, and left caudal ACC, which are associated with modulation of pain sensations, are less activated, and the pain rating is lower in alexithymics [26]. Yet, the greater activity in the insula indicates a stronger response in the representation of the internal physiologic state in alexithymics [26].

There are overlapping characteristics of deficits in emotional experience and expression between posttraumatic stress disorder (PTSD) and alexithymia [7,8]. Frewen et al. investigated functional neural responses to trauma script imagery associated with severity of alexithymia in a subsample of 26 individuals with PTSD [71]. The TAS-20 score was correlated with increased activity in the right posterior insula and ventral posterior cingulate and decreased activity in the bilateral ventral ACC, ventromedial PFC (vmPFC), anterior insula, and right inferior frontal cortex [71]. Correlation between alexithymic score and right insular activation might be associated with centrally represented body-state mapping of sympathetic arousal, coupled with reduced executive-regulatory cognitive-affective control via the ventral ACC, medial (mPFC), and right inferior frontal cortex $[43,45,65,66]$.

Both Moriguchi's [22] and Frewen's [70] studies mentioned above provide further evidence that alexithymia is associated with greater activation in the insular cortex representing interoceptive arousal in response to the internal body-state condition. Interestingly, a recent study measured empathic brain responses in subjects with autism spectrum disorder and control subjects, in which participants anticipated their partner's pain, which was indicated by an anticipatory cue (colored arrow) [72]. Activity in the anterior insula during the task was negatively correlated with individual differences in TAS score in both subjects with autism spectrum disorder and control subjects [72]. It is quite an interesting phenomenon that real pain and pictures of painful stimuli or a traumatic event induce hyperactivity in the posterior insula, whereas imagination of a partner's pain provokes less activation in the anterior insula associated with alexithymia. This gradation of activity in the insular cortex accompanying the gradation of the real level of pain appears to explain alexithymic features remarkably. With regard to pain experience, alexithymics may experience pain more strongly at a primitive perceptual level and may not be able to experience pain at a more abstract, conceptual, formal, operational stage. This phenomenon matches the theory of deficient affect development in alexithymia $[7,11]$.

\section{Interoceptive awareness}

Another notable finding with respect to interoception in alexithymia is interoceptive awareness. Interoceptive awareness tasks measure the ability to perceive (count) one's heartbeat and represents general sensitivity to visceral processes [73]. Neuroimaging studies have revealed that the anterior insular cortex is of relevance for interoceptive awareness and the rerepresentation and integration of interoceptive bodily signals with higher-order and emotional processes [66]. Interestingly, the heartbeat perception score was inversely correlated with alexithymia in 155 university student subjects, suggesting that alexithymics have less interoceptive awareness [73]. This finding appears to be inconsistent with our results, in which alexithymics show hyperinteroceptive arousal during visceral pain. The discrepancy may be owing to the nature of the tasks. The arousal level of interoception is different; the heartbeat detection task appears to be less arousing than the visceral pain condition. The heartbeat detection task measures attention processing of interoception more and visceral pain processing measures the perception process more. Alexithymics may feel interoceptive arousal more when the bodily state is strongly aroused, or there might be some uncoupling between the attention system and the perception system to bodily signals. Whether activity in the anterior or posterior insular cortex in alexithymia is less or more during the heartbeat detection task should be examined in the future.

\section{Chronic pain and alexithymia}

Although our results provide evidence that alexithymics may be hypersensitive to interoceptive arousal, recent reports suggest that alexithymia may be related mainly to the affective-rather than the sensory-dimension of chronic pain $[20,55]$. However, it has been reported that alexithymia is associated with responses to sustained, high-intensity stimuli (pain tolerance level) but not to low-intensity stimuli (pain threshold level) in fibromyalgia [74]. Enhanced sensitivity to unpleasant sensations in 
alexithymic individuals may become more pronounced during high-intensity stimulation or after prolonged pain stimulation [56]. Association between alexithymia and chronic pain syndromes cannot be explained simply by hypersensitivity to sensory components of painful conditions. Neuroimaging data indicate not only hyperactivity in visceral perception areas, such as the insular cortex, but also hypoactivity in pain-processing, regulatory areas such as prefrontal cortex. Lack of an emotional regulation system might cause hypersensitivity to aversive bodily sensations and prolonged, pain-related affective reactions such as distress. This is a possible mechanism underlying the association between alexithymia and chronic pain.

\section{Decision-making process based on emotion-guided evaluation in alexithymia}

The regulation of emotions has been conceptualized as an integrative, interactional process among the three domains of the emotional response system and with the environment. Affect regulation is a process involving reciprocal interactions between the neurophysiologic, motor-expressive, and cognitive-experiential domains of the emotional response system. Because activation in any one response domain alters or modulates activation in the other two domains, all three domains are involved in the regulation of emotion. Clearly, social bonds, language, dreams, fantasy, play, crying, smiling, and defense mechanisms all play roles in emotional regulation, as does afferent feedback from peripheral autonomic activity and the musculoskeletal system [7]. Affects have important organizing, motivating, and adaptive functions.

Studies that we discuss in this article focus on one domain of the emotional response system, such as response to facial expression for the cognitive-experiential domain or response to visceral pain for the neurophysiologic domain. The true problem in alexithymia, affect dysregulation, is not a simple deficit in one domain of the emotional response system. It is a dysfunction in the interactions among each domain and a dysfunction in the interaction between the emotional response system and the environment. In a third neuroimaging study on alexithymia, we investigated the ability to use emotional signals to guide behavior in social situations.

The Iowa gambling task (IGT) was developed to assess how the decision-making process is influenced by emotionally biased signals, from the observation of patients with damage to the vmPFC [75]. Such decision-making as seen in the IGT presupposes the theory of "somatic markers," as developed by Damasio [76]. This theory argues that optimal decision-making is not simply the result of rationally, cognitively calculated categorization of gains and losses but is also based on good or bad affective reactions and emotionally guided evaluation.
The decision-making deficits found after vmPFC damage owe to an inability to use emotion-based biasing signals generated from the body (somatic markers) when appraising different response options. The reasoning is influenced by crude biasing signals arising from the neural machinery that underlies emotion.

Participants with alexithymia fail to learn an advantageous decision-making strategy, with performance differing significantly from the nonalexithymic group in the last IGT trial [76]. Comparing performance on the IGT and the control task, both groups show brain activation in areas associated with IGT including the PFC, supplementary motor area, inferior parietal lobe, fusiform gyrus, and cerebellum [77]. In addition, nonalexithymics show activation in the bilateral insula and ACC, which have been proposed to house some of the neural substrates of somatic markers. However, alexithymics demonstrate no activation in the insula or ACC but do show activation in the caudate. Comparison between alexithymics and nonalexithymics reveals that alexithymics show lower $\mathrm{rCBF}$ in the vmPFC (Brodmann area [BA] 10) and higher $\mathrm{rCBF}$ in the caudate and occipital areas during a learning phase of IGT trials [24].

In the context of IGT-related processing, BA10 activity may be associated with the use of internal signals accompanying affective evaluation of stimuli, which is crucial for successful decision-making. The difference in BA10 activity indicates that subjects with alexithymia cannot use brain functions related to emotion-based biasing signals. However, the function of the caudate is to regulate or control impulsivity and disinhibition, and its activity during decision-making has been suggested to be a marker of risk attitude. Individuals with alexithymia may work on the IGT impulsively rather than by using emotion-based signals. This IGT study suggests that individuals with alexithymia may be unable to use feelings to guide their behavior appropriately, in addition to being unable to recognize emotions, which supports previous findings.

There is some behavioral data to support our findings. Undergraduate students $(\mathrm{N}=326)$ performed the standard IGT and a version in which cumulative financial feedback was obscured [78]. Standard learning on the IGT was observed for those scoring low for alexithymia. Higher alexithymia scores were associated with an attenuated learning rate on the IGT, especially under conditions of reduced cognitive information. Those scoring high for alexithymia showed a pattern of response choices that indicated increased risk-taking toward the end of the task [78]. The cognitive skills required to effectively monitor and self-regulate emotions are encompassed in a construct known as emotional intelligence. The emotional intelligence score is inversely correlated with the TAS-20 score [78]. Patients with lesions in 
structures in the somatic marker circuitry show significantly lower emotional intelligence (high TAS-20 score) and poorer judgment in decision-making, as well as disturbances in social functioning, compared to those with lesions in structures outside the somatic marker circuitry [79].

Taken together, alexithymic individuals may be unable to use emotion for flexible cognitive regulation. Thus, there may be uncoupling or dysfunction in the interaction of the aspects of the emotional response system in alexithymia.

\section{Cognitive emotional development model and alexithymia} Lane and Schwartz [80] conceptualized a cognitivedevelopment model for understanding the organization of emotional experience (Figure 1A). In this model, the experience of emotion is hypothesized to undergo structural transformation in a hierarchical developmental sequence of progressive differentiation and integration. There are five levels of emotional organization and awareness in the model: awareness of physical sensations (level 1 ), action tendencies (level 2), single emotions (level 3), blends of emotions (level 4), and blends of blends of emotional experience (level 5, the capacity to appreciate complexity in the experiences of self and others). The levels are hierarchically related in that functioning at each level adds to and modifies function of the previous levels but does not eliminate them.

Each level of organization corresponds to an identifiable neurobiologic state. Levels 1 and 2 involve implicit processes that are automatic, modular, and cognitively impenetrable. Subcortical structures may participate in the automatic generation of emotional responses associated with absent or diffusely undifferentiated awareness. Neural substrates of level 1 may include the thalamus and hypothalamus (diencephalon) and brainstem. At level 2, the sensorimotor enactive level, the thalamus, amygdala, and ventral striatum would be involved in association with crude distinctions between globally positive and globally negative states. Emotions at level 2 are represented in actions, and the basal ganglia participate in the automatic behavioral displays of emotional expression. Orbitofrontal cortex activity appears to be associated with the perception of bodily sensations that bias behavior. For example, orbitofrontal cortex activity affects behavior by overriding automatic processes in the amygdala.

Levels 3 through 5 involve explicit processes that are influenced by higher cognitive processes, including prior knowledge. They are hypothesized to be mediated by the structures at levels 1 and 2 and by paralimbic structures, including the ACC and insula, and the mPFC. The rostral ACC/mPFC appears to be necessary for the representation of emotion used in conscious cognition. The process of representational redescription is mediated, in part, by structures involved in explicit processing at levels 3 through 5 . Because these structures are not uniquely devoted to the processing of emotion, their emotionrelated functions are cognitively penetrable. The level of emotional awareness of a given individual may be a function of the degree to which these structures are or are not devoted to processing of emotional information from the internal and external worlds. The hierarchical nature of this anatomic model is parallel to the hierarchical structure of the psychologic model.

\section{Conclusions}

We have conducted three brain imaging studies on alexithymia. The first study displayed weakness of cognitive emotional processing, with less activation in the dACC and anterior insula during the viewing of angry facial
A
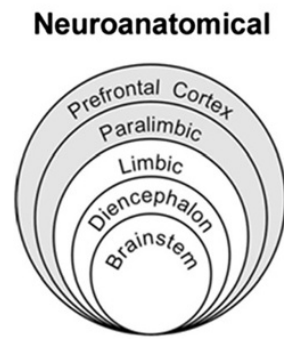
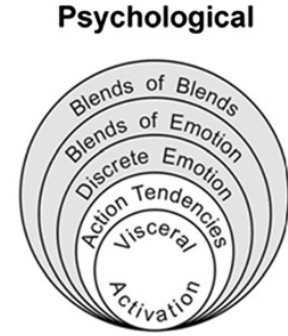

B

\section{Alexithymic brain}

Socio-affective processing $\downarrow$

Anterior insula $\downarrow[27,37,48]$ Amygdala $\downarrow[25-27,30,46-48]$ ACC $\downarrow[17,18,21,46,70]$ 个 $[17,22,26]$

\section{Emotional guiding decision making -inflexible vmPFC $\downarrow[20]$ Caudate $\uparrow[20]$}

\section{Interoceptive arousal $\uparrow$}

Posterior insula $\uparrow[19,22,70]$

Figure 1 A: Schema for the five levels of emotional awareness model of Lane and Schwartz [80], Parallels in the hierarchical organization of emotional experience and its neural substrates. The shell structure is intended to convey that each succeeding level adds to and modulates lower levels but does not replace them. Although each model contains five levels, a one-to-one correspondence between each level in the psychological and neuroanatomical models is not intended. Lower levels with white background correspond to implicit processes. Higher levels with gray background correspond to explicit processes. B: Neural machinery characterized by neuroimaging studies on alexithymia. Alexithymics showed weak responses in structures of highly cognitive stages, such as the capacity to feel other's emotional reactions (socioaffective processing), and stronger responses in primitive stages such as hypersensitivity to visceral pain (interoceptive aroursal). Consequently alexithymics experience inflexible cognitive regulation, owing to impairment of the emotional guiding system. ACC $=$ Anterior cingulate cortex; vmPFC = ventromedial prefrontal cortex. 
expressions. This may represent less function in cognitive mature stages such as levels 3 through 5 . The second study showed stronger awareness of visceral sensation in alexithymics, with greater activity in the brainstem, posterior insula, and rostral ACC, accompanying stronger autonomic responses. This indicates stronger responses at primitive stages such as levels 1 and 2 . The third study demonstrated greater activation in the caudate (basal ganglia) and less activation in the $\mathrm{MPFC}$ in alexithymics during the IGT, which may represent impairment of the interactive, integrative process among the five levels. Thus alexithymics show weak responses in structures necessary for the representation of emotion used in conscious cognition and stronger responses at levels focused on action. Consequently, alexithymics experience inflexible cognitive regulation, owing to impairment of the emotionguiding system.

The problem in alexithymia has been posited to be deficient development of the cognitive mature stage, which allows some rudimentary form of emotional experience, such as high arousal of bodily sensations. Our neuroimaging studies provide neural evidence for this hypothesis (Figure 1B). A mature emotional system might have a role in alleviating physical sensations and in guiding flexible behavior. Deficient emotional development in alexithymia might lead to hypersensitivity to bodily sensations and unhealthy behaviors, which may be a possible link between alexithymia and psychosomatic disorders.

Alexithymia has been linked with psychiatric conditions, including high-functioning autism/Asperger syndrome and depression, in addition to somatoform disorders [81]. Asperger's syndrome is characterised by core disturbances in speech and language and problems with affective interaction and has been indicated to have an overlapping character with alexithymia [82,83]. In terms of socioaffective processing, Asperger's syndrome has similar features to alexithymia. Patients with Asperger's syndrome showed failure to use information from faces, such as facial affect, eye gaze and facial expression [84], and anomalous activity in the amygdala, fusiform gyrus, and superior temporal gyrus during facial expression recognition [85]. As mentioned above, during anticipation of the partner's pain, the strength of the signal in the anterior insula was predictive of the degree of alexithymia in both autistic and control groups [72]. However, there was no difference between autistic and control groups after accounting for alexithymia, suggesting that the empathy deficits observed in autism may be due to the large comorbidity between alexithymic traits and autism, rather than representing a necessary feature of the social impairments in autism [72]. In contrast to the reactivity in the amygdala in individuals with alexithymia, many studies have demonstrated hyperactivity in the amygdala in response to negative facial expressions [86-89] and in response to other negative emotional stimuli $[90,91]$ in depressed subjects. Recent studies in adolescents with depression [92,93] and at risk for depression [94] have confirmed that amygdala hyper-responsivity is present in the early stages of illness. Alexithymia has been reported to be associated strongly with depression in both general and clinical populations [6]. It has also been reported that TAS scores increased during depression and decreased after remission [95] and closely linked to concurrent depressive symptoms [96]. However, TAS scores did not predict diagnoses of major depression in a 7-year follow-up study [96]. Co-mobility of depressive disorder cannot explain the neurobiological features of alexithymia. On the other hand, recent study has shown less activity in somatoform patients in the emotional brain circuit, including the bilateral parahippocampal gyrus, the left amygdala, the left superior temporal gyrus, and the insula, during facial emotion recognition. Somatoform patients exhibited increased alexithymia scores (TAS-20) and elevated depression scores (BDI) [97]. Therefore, their neurobiological features to emotional stimuli were more similar to alexithymia.

This article considers the role of emotion in the development of physical symptoms and discusses a possible pathway that we have identified in our neuroimaging studies linking alexithymia with psychosomatic disorders. The Alexithymia construct should be further investigated, not only as psychological problems in affect regulation but also from the viewpoint of an association with physiological symptoms.

Competing interests

The authors declare that they have no competing interests.

Authors' contributions

MK: Study concept and design; interpretation of data; drafting of the manuscript; critical revision of the manuscript for important intellectual content. SF: critical revision of the manuscript for important intellectual content. Both authors read and approved the final manuscript.

Received: 26 March 2012 Accepted: 13 December 2012

Published: 9 January 2013

\section{References}

1. Sifneos PE: The prevalence of 'alexihtymic' characteristic mechanisms in psychosomatic patients. Psychother Psychosom 1973, 21:133-136.

2. Nemiah JC, Freyberger H, Sifneos PE: Alexithymia: a view of the psychosomatic process. Lodon: Butterworths; 1976.

3. Bagby RM, Paker JDA, Talor GJ: The twenty-item Toronto alexithymia scale I.Item selection and cross-validation of the factor structure. J Psychosom Res 1994, 38:33-40.

4. Bagby RM, Talor GJ, Paker JDA: The twenty-item toronto alexithymia ScalellConvergent, discriminant, and concurrent validity. J Psychosom Res 1994, 38:23-32.

5. Lumley MA, Neely LC, Burger AJ: The assessment of alexithymia in medical settings: implications for understanding and treating health problems. J Pers Assess 2007, 89:230-246.

6. Taylor GJ, Bagby RM: New trends in alexithymia research. Psychother Psychosom 2004, 73:68-77.

7. Taylor GJ, Bagby RM, Parker JDA: Disorder of affect regulation: Alexithymia in medical and psychiatric illness. New York: Cambridge Univ Press; 1997.

8. Lane RD, Ahern GL, Schwartz GE, Kaszniak AW: Is alexithymia the emotional equivalent of blindsight? Biol Psychiatry 1997, 42:834-844. 
9. Kooiman CG, Spinhoven P, Trijsburg RW: The assessment of alexithymia: a critical review of the literature and a psychometric study of the toronto alexithymia scale-20. J Psychosom Res 2002, 53:1083-1090.

10. Bagby RM, Taylor GJ, Parker JD, Dickens SE: The development of the toronto structured interview for alexithymia: item selection, factor structure, reliability and concurrent validity. Psychother Psychosom 2006, 75:25-39.

11. Lane RD, Schwartz GE: Levels of emotional awareness - a cognitivedevelopmental theory and its application to psychopathology. Am J Psychiatry 1987, 144:133-143.

12. Taylor GJ: Recent developments in alexithymia theory and research. Can J Psychiatry 2000, 45:134-142.

13. Lumley MA, Radcliffe AM, Macklem DJ, Mosley-Williams A, Leisen JC, Huffman JL, D'Souza PJ, Gillis ME, Meyer TM, Kraft CA, Rapport LJ: Alexithymia and pain in three chronic pain samples: comparing caucasians and african americans. Pain Med 2005, 6:251-261.

14. Porcelli P, De Carne M, Todarello O: Prediction of treatment outcome of patients with functional gastrointestinal disorders by the diagnostic criteria for psychosomatic research. Psychother Psychosom 2004, 73:166-173

15. Grabe HJ, Schwahn C, Barnow S, Spitzer C, John U, Freyberger HJ, Schminke U, Felix S, Volzke H: Alexithymia, hypertension, and subclinical atherosclerosis in the general population. J Psychosom Res 2010, 68:139-147.

16. Kauhanen J, Kaplan GA, Cohen RD, Julkunen J, Salonen JT: Alexithymia and risk of death in middle-aged men. J Psychosom Res 1996. 41:541-549.

17. Tolmunen T, Lehto SM, Heliste M, Kurl S, Kauhanen J: Alexithymia is associated with increased cardiovascular mortality in middle-aged finnish men. Psychosom Med 2010, 72:187-191.

18. Taylor GJ, Bagby RM, Parker JD: The alexithymia construct. A potential paradigm for psychosomatic medicine. Psychosomatics 1991, 32:153-164

19. Dragos D, Tanasescu MD: The critical role of psychosomatics in promoting a new perspective upon health and disease. J Med Life 2009, 2:343-349

20. Lumley MA, Stettner L, Wehmer F: How are alexithymia and physical illness linked? a review and critique of pathways. J Psychosom Res 1996, 41:505-518.

21. Berthoz S, Artiges E, Van De Moortele PF, Poline JB, Rouquette S, Consoli $S M$, Martinot JL: Effect of impaired recognition and expression of emotions on frontocingulate cortices: an fMRI study of men with alexithymia. Am J Psychiatry 2002, 159:961-967.

22. Kano M, Fukudo S, Gyoba J, Kamachi M, Tagawa M, Mochizuki H, Itoh M, Hongo $M$, Yanai $K$ : Specific brain processing of facial expressions in people with alexithymia: an H2 150-PET study. Brain 2003, 126:1474-1484.

23. Kano M, Hamaguchi T, Itoh M, Yanai K, Fukudo S: Correlation between alexithymia and hypersensitivity to visceral stimulation in human. Pain 2007, 132:252-263.

24. Kano M, Ito M, Fukudo S: Neural substrates of decision making as measured with the iowa gambling task in men with alexithymia. Psychosom Med 2011, 73:588-597.

25. Mantani T, Okamoto Y, Shirao N, Okada G, Yamawaki S: Reduced activation of posterior cingulate cortex during imagery in subjects with high degrees of alexithymia: a functional magnetic resonance imaging study. Biol Psychiatry 2005, 57:982-990.

26. Moriguchi Y, Decety J, Ohnishi T, Maeda M, Mori T, Nemoto K, Matsuda H, Komaki G: Empathy and judging other's pain: an fMRI study of alexithymia. Cereb Cortex 2007, 17:2223-2234.

27. Moriguchi Y, Ohnishi T, Lane RD, Maeda M, Mori T, Nemoto K, Matsuda H, Komaki G: Impaired self-awareness and theory of mind: an fMRI study of mentalizing in alexithymia. Neuroimage 2006, 32:1472-1482.

28. Moriguchi Y, Ohnishi T, Decety J, Hirakata M, Maeda M, Matsuda H, Komak $\mathrm{G}$ : The human mirror neuron system in a population with deficient selfawareness: an fMRI study in alexithymia. Hum Brain Mapp 2009, 30:2063-2076

29. Zotev V, Krueger F, Phillips R, Alvarez RP, Simmons WK, Bellgowan P, Drevets WC, Bodurka J: Self-regulation of amygdala activation using realtime FMRI neurofeedback. PLoS One 2011, 6:e24522.

30. Pouga L, Berthoz S, de Gelder B, Grezes J: Individual differences in socioaffective skills influence the neural bases of fear processing: the case of alexithymia. Hum Brain Mapp 2010, 31:1469-1481.
31. Reker M, Ohrmann P, Rauch AV, Kugel H, Bauer J, Dannlowski U, Arolt V, Heindel W, Suslow T: Individual differences in alexithymia and brain response to masked emotion faces. Cortex 2010, 46:658-667.

32. Heinzel A, Schafer R, Muller HW, Schieffer A, Ingenhag A, Eickhoff SB, Northoff G, Franz M, Hautzel $H$ : Increased activation of the supragenual anterior cingulate cortex during visual emotional processing in male subjects with high degrees of alexithymia: an event-related fMRI study. Psychother Psychosom 2010, 79:363-370.

33. Swart M, Bruggeman R, Laroi F, Alizadeh BZ, Kema I, Kortekaas R, Wiersma D, Aleman A: COMT Val158Met polymorphism, verbalizing of emotion and activation of affective brain systems. Neuroimage 2011, 55:338-344.

34. Kugel H, Eichmann M, Dannlowski U, Ohrmann P, Bauer J, Arolt V, Heinde W, Suslow T: Alexithymic features and automatic amygdala reactivity to facial emotion. Neurosci Lett 2008, 435:40-44.

35. Frewen PA, Pain C, Dozois DJ, Lanius RA: Alexithymia in PTSD: psychometric and FMRI studies. Ann N Y Acad Sci 2006, 1071:397-400.

36. Koponen S, Taiminen T, Honkalampi K, Joukamaa M, Viinamaki H, Kurki T, Portin R, Himanen L, Isoniemi H, Hinkka S, Tenovuo O: Alexithymia after traumatic brain injury: its relation to magnetic resonance imaging findings and psychiatric disorders. Psychosom Med 2005, 67:807-812.

37. Lane R, Sechrest L, Reidel R, Brown V, Kaszniak A, Schwartz G: Alexithymia and nonverbal emotion processing deficits. Psychosom Med 1995, 57:84.

38. Lane RD, Sechrest L, Riedel R, Shapiro DE, Kaszniak AW: Pervasive emotion recognition deficit common to alexithymia and the repressive coping style. Psychosom Med 2000, 62:492-501.

39. Vermeulen $\mathrm{N}$, Luminet $\mathrm{O}$, Corneille O: Alexithymia and the autonomatic processing of affective information: evidence from the affective priming paradigm. Cognitition and emotion 2006, 20:64-91.

40. Adolphs R: Neural systems for recognizing emotion. Curr Opin Neurobiol 2002, 12:169-177.

41. McRae K, Reiman EM, Fort CL, Chen K, Lane RD: Association between trait emotional awareness and dorsal anterior cingulate activity during emotion is arousal-dependent. Neuroimage 2008, 41:648-655.

42. Lane RD, Reiman EM, Axelrod B, Yun LS, Holmes A, Schwartz GE: Neural correlates of levels of emotional awareness. Evidence of an interaction between emotion and attention in the anterior cingulate cortex. J Cogn Neurosci 1998, 10:525-535.

43. Craig AD: How do you feel? interoception: the sense of the physiological condition of the body. Nat Rev Neurosci 2002, 3:655-666.

44. Craig AD: How do you feel-now? the anterior insula and human awareness. Nat Rev Neurosci 2009, 10:59-70.

45. Critchley HD: Neural mechanisms of autonomic, affective, and cognitive integration. J Comp Neurol 2005, 493:154-166.

46. Critchley HD: Psychophysiology of neural, cognitive and affective integration: fMRI and autonomic indicants. Int J Psychophysiol 2009, 73:88-94.

47. Leweke F, Stark R, Milch W, Kurth R, Schienle A, Kirsch P, Stingl M, Reimer C, Vaitl D: Patterns of neuronal activity related to emotional stimulation in alexithymia. Psychother Psychosom Med Psychol 2004, 54:437-444.

48. Li CS, Sinha R: Alexithymia and stress-induced brain activation in cocainedependent men and women. J Psychiatry Neurosci 2006, 31:115-121.

49. Silani G, Bird G, Brindley R, Singer T, Frith C, Frith U: Levels of emotional awareness and autism: an fMRI study. Soc Neurosci 2008, 3:97-112.

50. LeDoux J: The emotional brain, fear, and the amygdala. Cell Mol Neurobiol 2003, 23:727-738

51. Davis $M$, Whalen PJ: The amygdala: vigilance and emotion. Mol Psychiatry 2001, 6:13-34

52. Meriau K, Wartenburger I, Kazzer P, Prehn K, Lammers CH, van der Meer E, Villringer A, Heekeren HR: A neural network reflecting individual differences in cognitive processing of emotions during perceptual decision making. Neuroimage 2006, 33:1016-1027.

53. Kreitler S, Niv D: Pain and alexithymia: the nature of a relation. Pain Clinic 2001, 13:13-38.

54. Glaros AG, Lumley MA: Alexithymia and pain in temporomandibular disorder. J Psychosom Res 2005, 59:85-88.

55. Lumley MA, Smith JA, Longo DJ: The relationship of alexithymia to pain severity and impairment among patients with chronic myofascial pain: comparisons with self-efficacy, catastrophizing, and depression. J Psychosom Res 2002, 53:823-830.

56. Nyklicek I, Vingerhoets AJ: Alexithymia is associated with low tolerance to experimental painful stimulation. Pain 2000, 85:471-475. 
57. Putterman E, Byrne N, Ditto B: Alexithymia and symptom reporting following blood donation. Psychosom Med 2001, 63:138-139.

58. Sivik T: Alexithymia and hypersensitivity to touch and palpation. Integr Physiol Behav Sci 1993, 28:130-136.

59. Nakao M, Barsky AJ, Kumano H, Kuboki T: Relationship between somatosensory amplification and alexithymia in a Japanese psychosomatic clinic. Psychosomatics 2002, 43:55-60.

60. Porcelli P, Taylor GJ, Bagby RM, De Carne M: Alexithymia and functional gastrointestinal disorders. A comparison with inflammatory bowel disease. Psychother Psychosom 1999, 68:263-269.

61. Porcelli P, Bagby RM, Taylor GJ, De Carne M, Leandro G, Todarello O: Alexithymia as predictor of treatment outcome in patients with functional gastrointestinal disorders. Psychosom Med 2003, 65:911-918.

62. Tillisch K, Mayer EA, Labus JS: Quantitative meta-analysis identifies brain regions activated during rectal distension in irritable bowel syndrome. Gastroenterology 2011, 140:91-100.

63. Derbyshire SW: A systematic review of neuroimaging data during visceral stimulation. Am J Gastroenterol 2003, 98:12-20.

64. Mayer EA, Naliboff BD, Craig AD: Neuroimaging of the brain-gut axis: from basic understanding to treatment of functional Gl disorders. Gastroenterology 2006, 131:1925-1942.

65. Craig AD: Interoception: the sense of the physiological condition of the body. Curr Opin Neurobiol 2003, 13:500-505.

66. Critchley HD, Wiens S, Rotshtein P, Ohman A, Dolan RJ: Neural systems supporting interoceptive awareness. Nat Neurosci 2004, 7:189-195.

67. Vogt BA: Pain and emotion interactions in subregions of the cingulate gyrus. Nat Rev Neurosci 2005, 6:533-544.

68. Neafsey EJ, Terreberry RR, Hurley KM, Ruit KG, Frysztak RJ: Anterior cingulate cortex in rodents: Connections, visceral control functions, and implications for emotion, in Neurobiology of Cingulate Cortex and Limbic Thalamus. Boston: Birkhauser; 1993.

69. Barbas $\mathrm{H}$ : Proceeding of the human cerebral cortex: from gene to structure and function. Brain Res Bull 2000, 52:319-330.

70. Dunckley P, Wise RG, Fairhurst M, Hobden P, Aziz Q, Chang L, Tracey I: A comparison of visceral and somatic pain processing in the human brainstem using functional magnetic resonance imaging. J Neurosci 2005, 25:7333-7341.

71. Frewen PA, Lanius RA, Dozois DJ, Neufeld RW, Pain C, Hopper JW, Densmore M, Stevens TK: Clinical and neural correlates of alexithymia in posttraumatic stress disorder. J Abnorm Psychol 2008, 117:171-181.

72. Bird G, Silani G, Brindley R, White S, Frith U, Singer T: Empathic brain responses in insula are modulated by levels of alexithymia but not autism. Brain 2010, 133:1515-1525.

73. Herbert BM, Herbert C, Pollatos O: On the Relationship Between Interoceptive Awareness and Alexithymia: Is Interoceptive Awareness Related to Emotional Awareness? J Pers 2011, 79:1149-1175.

74. Huber A, Suman AL, Biasi G, Carli G: Alexithymia in fibromyalgia syndrome: associations with ongoing pain, experimental pain sensitivity and illness behavior. J Psychosom Res 2009, 66:425-433.

75. Bechara A, Damasio AR, Damasio H, Anderson SW: Insensitivity to Future Consequences Following Damage to Human Prefrontal Cortex. Cognition 1994, 50:7-15.

76. Damasio AR: Descartes' Error: Emotion, Reason, and the Human Brain. New York: Putnum; 1994.

77. Li XR, Lu ZL, D'Argembeau A, Ng M, Bechara A: The lowa Gambling Task in fMRI Images. Hum Brain Mapp 2010, 31:410-423.

78. Ferguson E, Bibby PA, Rosamond S, O'Grady C, Parcell A, Amos C, McCutcheon C, O'Carroll R: Alexithymia, Cumulative Feedback, and Differential Response Patterns on the lowa Gambling Task. J Pers 2009, 77:883-902

79. Bar-On R, Tranel D, Denburg NL, Bechara A: Exploring the neurological substrate of emotional and social intelligence. Brain 2003, 126:1790-1800.

80. Lane RD: Neural Correlates of Conscious Emotional Experience. In Cognitive neuroscience of emotion. Edited by Lane RD, Nadel L. USA: Oxford University Press, Inc; 1999:345-370.

81. Bankier B, Aigner M, Bach M: Alexithymia in DSM-IV disorder: comparative evaluation of somatoform disorder, panic disorder, obsessive-compulsive disorder, and depression. Psychosomatics 2001, 42:235-240.

82. Fitzgerald M, Bellgrove MA: The overlap between alexithymia and Asperger's syndrome. J Autism Dev Disord 2006, 36:573-576.
83. Hill EL, Berthoz S: Response to "Letter to the Editor: The overlap between alexithymia and Asperger's syndrome", Fitzgerald and Bellgrove, Journal of Autism and Developmental Disorders, 36(4). J Autism Dev Disord 2006, 36:1143-1145

84. Verhoeven JS, De Cock P, Lagae L, Sunaert S: Neuroimaging of autism. Neuroradiology 2010, 52:3-14

85. Harms MB, Martin A, Wallace GL: Facial emotion recognition in autism spectrum disorders: a review of behavioral and neuroimaging studies. Neuropsychol Rev 2010, 20:290-322

86. Fu CH, Williams SC, Cleare AJ, Brammer MJ, Walsh ND, Kim J, Andrew CM, Pich EM, Williams PM, Reed $\sqcup$, et al: Attenuation of the neural response to sad faces in major depression by antidepressant treatment: a prospective, event-related functional magnetic resonance imaging study. Arch Gen Psychiatry 2004, 61:877-889

87. Surguladze S, Brammer MJ, Keedwell P, Giampietro V, Young AW, Travis MJ, Williams SC, Phillips ML: A differential pattern of neural response toward sad versus happy facial expressions in major depressive disorder. Biol Psychiatry 2005, 57:201-209.

88. Fales CL, Barch DM, Rundle MM, Mintun MA, Snyder AZ, Cohen JD, Mathews J, Sheline Yl: Altered emotional interference processing in affective and cognitive-control brain circuitry in major depression. Biol Psychiatry 2008, 63:377-384.

89. Sheline YI, Barch DM, Donnelly JM, Ollinger JM, Snyder AZ, Mintun MA: Increased amygdala response to masked emotional faces in depressed subjects resolves with antidepressant treatment: an fMRI study. Biol Psychiatry 2001, 50:651-658.

90. Siegle GJ, Thompson W, Carter CS, Steinhauer SR, Thase ME: Increased amygdala and decreased dorsolateral prefrontal BOLD responses in unipolar depression: related and independent features. Biol Psychiatry 2007, 61:198-209.

91. Abler B, Erk S, Herwig U, Walter H: Anticipation of aversive stimuli activates extended amygdala in unipolar depression. J Psychiatr Res 2007, 41:511-522.

92. Roberson-Nay R, McClure EB, Monk CS, Nelson EE, Guyer AE, Fromm SJ, Charney DS, Leibenluft E, Blair J, Ernst M, Pine DS: Increased amygdala activity during successful memory encoding in adolescent major depressive disorder: An FMRI study. Biol Psychiatry 2006, 60:966-973.

93. Beesdo K, Lau JY, Guyer AE, McClure-Tone EB, Monk CS, Nelson EE, Fromm SJ, Goldwin MA, Wittchen HU, Leibenluft E, et al: Common and distinct amygdala-function perturbations in depressed vs anxious adolescents. Arch Gen Psychiatry 2009, 66:275-285

94. Monk CS, Klein RG, Telzer EH, Schroth EA, Mannuzza S, Moulton JL 3rd, Guardino M, Masten CL, McClure-Tone EB, Fromm S, et al: Amygdala and nucleus accumbens activation to emotional facial expressions in children and adolescents at risk for major depression. Am J Psychiatry 2008, 165:90-98.

95. Marchesi C, Bertoni S, Cantoni A, Maggini C: Is alexithymia a personality trait increasing the risk of depression? A prospective study evaluating alexithymia before, during and after a depressive episode. Psychol Med 2008, 38:1717-1722

96. Honkalampi K, Koivumaa-Honkanen H, Lehto SM, Hintikka J, Haatainen K, Rissanen T, Viinamaki $\mathrm{H}$ : Is alexithymia a risk factor for major depression, personality disorder, or alcohol use disorders? A prospective populationbased study. J Psychosom Res 2010, 68:269-273.

97. de Greck M, Scheidt L, Bolter AF, Frommer J, Ulrich C, Stockum E, Enzi B, Tempelmann C, Hoffmann T, Han S, Northoff G: Altered brain activity during emotional empathy in somatoform disorder. Hum Brain Mapp 2012, 33:2666-2685.

doi:10.1186/1751-0759-7-1

Cite this article as: Kano and Fukudo: The alexithymic brain: the neural pathways linking alexithymia to physical disorders. BioPsychoSocial Medicine 2013 7:1. 